\title{
Time-Resolved Visualization of Instability Waves in a Hypersonic Boundary Layer
}

\author{
S. J. Laurence ${ }^{1}$, A. Wagner ${ }^{1}$, and K. Hannemann ${ }^{2}$, \\ German Aerospace Center, Göttingen D 37073, Germany \\ V. Wartemann ${ }^{3}$ and H. Lüdeke ${ }^{4}$ \\ German Aerospace Center, Braunschweig D 38108, Germany \\ and \\ H. Tanno ${ }^{5}$ and K. Ito $^{6}$ \\ Japanese Aerospace Exploration Agency, Kakuda, Miyagi, 981-1525, Japan
}

\section{Introduction}

【AMINAR-turbulent transition in hypersonic boundary layers remains a challenging subject. This is especially true of the hypervelocity regime, in which an intriguing phenomenon is the possible damping of second-mode disturbances by chemical and vibrational nonequilibrium processes ${ }^{1,2}$. To generate flows with sufficiently high enthalpy to investigate such effects, the use of shock-tunnel facilities is necessary; furthermore, it is now generally accepted that direct measurements of the instability mechanisms active within the boundary layer, together with a characterization of the free-stream disturbance environment, are required, as simple measurements of transition locations can lead to ambiguous conclusions ${ }^{3,4}$. However, as difficult as the accurate measurement of instability waves in conventional hypersonic facilities can be, in shock tunnels it is appreciably more so. For identical unit Reynolds numbers, the higher stagnation temperature in a shock tunnel means that the dominant second-mode disturbances lie at even higher frequencies (typically hundreds of $\mathrm{kHz}$ or higher); moreover, because of the destructive testing environment, hot-wire techniques, a staple for instability measurements in conventional tunnels, cannot be used. Fast-response pressure transducers are an obvious alternative, but recent experiments ${ }^{5}$ have highlighted the challenging nature of interpreting data from mechanically-sensitive sensors in the high-noise

\footnotetext{
${ }^{1}$ Research Staff, Institute of Aerodynamics and Flow Technology, Spacecraft Department, Bunsenstraße 10. AIAA Member.

${ }^{2}$ Head, Spacecraft Department, Institute of Aerodynamics and Flow Technology, Bunsenstraße 10. AIAA Member.

${ }^{3}$ Research Staff, Institute of Aerodynamics and Flow Technology, Spacecraft Department, Lilienthalplatz 7.

${ }^{4}$ Research Staff, Institute of Aerodynamics and Flow Technology, Spacecraft Department, Lilienthalplatz 7. Senior AIAA Member.

${ }^{5}$ Associate Senior Researcher, Advanced Propulsion Research Group, 1 Koganesawa, Kimigaya.

${ }^{6}$ Head, High Enthalpy Flow Research Section, Advanced Propulsion Research Group, 1 Koganesawa, Kimigaya. AIAA Member
} 
environment of a shock tunnel, especially without accompanying stability computations. Measurements with recently developed atomic-layer thermopile (ALTP) heat-flux sensors show promise ${ }^{6}$, though their use has yet to be demonstrated in shock-tunnel facilities.

Single spark visualizations of instability waves in hypersonic boundary layers have been obtained by previous researchers $^{7-9}$ : the observed 'rope-like' waves typically have wavelengths approximately twice the boundary-layer thickness, and thus have been identified as second-mode disturbances. If quantitative information could be derived from high-speed sequences of such visualizations, however, the benefits are immediate: the measurements are nonintrusive; both temporal and extensive spatial information is obtained, including the ability to measure away from the model surface; and the spatial resolution, determined by the effective pixel resolution, is potentially higher than that available with other techniques (though for a given image resolution, some trade-off is required between the spatial resolution and the extent of the viewing area). However, to unambiguously resolve the periodic wave motion, the recording frequency must be at least as high as the wave frequency, requiring an extremely fast camera. A further disadvantage is that optical techniques are limited to line-of-sight measurements. However, the physical structure of second-mode disturbances in hypersonic boundary layers as determined by other researchers ${ }^{8,10}$, namely that they are two-dimensional oscillations primarily in density rather than velocity, suggests them to be good candidates for optical-based measurement.

This technical note is concerned with demonstrating the possibility of employing time-resolved Schlieren visualizations to determine the structural and propagation characteristics of second-mode instability waves within a hypersonic boundary layer. The result is particularly of note as the measurements were obtained in a shock-tunnel facility.

\section{Visualization Experiments}

Experiments were carried out in HEG (High Enthalpy shock tunnel Göttingen) of the German Aerospace Center. HEG is a free-piston-driven, reflected-shock wind tunnel capable of producing flows over a wide range of stagnation conditions for test durations of a few milliseconds. The model was a $1.1 \mathrm{~m}$ long, $7^{\circ}$ half-angle cone, having an 
interchangeable nose with a blunted tip radius of either 2.25 or $5 \mathrm{~mm}$; this model was previously employed for transition measurements in the JAXA HIEST shock tunnel ${ }^{5}$. The surface of the cone was instrumented with 136 coaxial thermocouples to determine approximate transition locations, and 8 PCB 132-A37 fast-response pressure transducers, grouped in pairs, to detect instability waves (two additional blind transducers were used to isolate mechanical vibrations). Visualization was by means of a dual-field lens Schlieren setup employing a $1000 \mathrm{~W}$ shortarc Xe lamp together with a Shimadzu HPV-1 camera, capable of recording 102 10-bit images at a fixed resolution of $312 \times 260$ pixels and at frame rates of up to $1 \mathrm{MHz}$. An extended light source was utilized to reduce the depth-offocus and minimize the influence of disturbances external to the boundary layer. Because of the desire to position the entire model within the core-flow region, it was only possible to visualize a fixed, $\sim 6 \mathrm{~cm}$ section of the cone surface, located approximately $83 \mathrm{~cm}$ from the cone tip (see Figure 1).

The experimental series was focused mainly on pressure measurements and the determination of transition locations; these are detailed in Ref. 11. However, in two runs the camera frame-rate was set sufficiently high and the transition location was such that it was possible to track laminar boundary-layer disturbances visually. Computed test conditions for these two experiments are given in Table 1 . The sequence in which the instability waves are most apparent (condition I), taken at a frame rate of $500 \mathrm{kHz}$ and with an exposure time of $0.5 \mu \mathrm{s}$, is shown in Figure 1. These images have been enhanced with an unsharp mask filter to accentuate detailed structures. Within the duration of the sequence, the development of a "rope-like" instability wave-packet is clearly visible (46-56 $\mu$ s in Figure 1). Figure 2 shows the time-developing scaled pixel intensities along a single horizontal row (corresponding to $y / \delta \approx 0.5$ ) in the form of an $x$-t diagram. The non-uniformities in illumination have been reduced by, at each time-step, the subtraction of first a reference flow-off image from the entire image and then the quadratic polynomial of best fit to the plotted row. The high-frequency noise has also been reduced through the application of a 5-point movingaverage filter. The development of the wave-packet in the images is reflected in the intensity profiles, and it is interesting to note that these profiles show a strong similarity to the hot-wire records obtained in Ref. 8, although, considering the completely different measurement methods employed, perhaps not too much should be read into this. 


\section{Determination of Disturbance Characteristics}

Quantitative information was extracted from the obtained images as follows. To determine the disturbance wavelength, power spectra of the pixel intensities along individual rows were calculated. An example is shown in Figure 3: for the image at $\mathrm{t}=54 \mu \mathrm{s}$, the spectral density is plotted against the normalized inverse wavelength, $\delta / \lambda$, for various scaled heights, $\mathrm{y} / \delta$, above the cone surface (where $\delta$ is the $99 \%$ boundary-layer thickness, based on velocity). Due to diffraction effects, the location of the cone surface could not be precisely determined; thus, these heights may be systematically offset by up to approximately $0.1 \delta$. The effective origin for $y / \delta$ here may also be uncertain in a more fundamental sense. The measurements of Kimmel et $\mathrm{a}^{10}$ have indicated that the spanwise extent of second-mode disturbances is limited to a few boundary-layer thicknesses; in this case, the apparent $y / \delta$ location will depend on the spanwise location of the disturbance, and the plotted values of $y / \delta$ may be offset in the negative direction. This limitation, intrinsic to non-focusing optical techniques employed with non-planar geometries, is discussed later in this note. The boundary-layer thickness here was calculated as $2.32 \mathrm{~mm}$ from a CFD simulation of the experiment using the FLOWer code ${ }^{12}$ (for comparison, the visualized boundary-layer thickness in the first image of Figure 1 is roughly $1.9 \mathrm{~mm}$, or 10 pixels). For second-mode disturbances, we would expect a peak in the power spectrum near $\delta / \lambda=0.5$, and such peaks are clearly present. The amplitude of the peak reaches a maximum near $\mathrm{y} / \delta=0.5$ and then quickly tapers off towards the boundary-layer edge. Measurements with hot-wire anemometers have shown second-mode disturbances to extend well past the boundary-layer edge ${ }^{8,13}$ : that such a phenomenon is not observed here may be related to the spanwise location of the disturbance, as mentioned above, or to the nature of the Schlieren measurements, discussed shortly. No higher harmonics are evident; the weaker peaks at longer wavelengths are likely due to low-frequency disturbances induced by tunnel noise or non-uniformities in the illumination profile. In the laminar spectra (e.g., those from the $2 \mu$ s image in Figure 1) only this low-frequency noise is present. Although the spectral profiles obtained here are certainly not as clean as those obtained by

researchers using hot-wire techniques ${ }^{8,13}$, they compare favorably with the spectra obtained from surface-mounted pressure transducers both in the present experiments and in Ref 5. Unfortunately, the plane of visualization here was too far from the pressure transducers for any comparison between visualization data and pressure traces to be made.

To measure the wave propagation speed, the displacement of the wave packet between images was determined through two-dimensional image correlation (only horizontal displacements were considered). Some subjectivity was 
introduced by the selection of the window corresponding to the wave packet, but the consistency of the values obtained suggested that this wasn't overly problematic. For various choices of image pairs, separated by up to $10 \mu \mathrm{s}$, propagation speeds of between 1910 and $1995 \mathrm{~m} / \mathrm{s}$ were obtained; a value of $1950 \pm 40 \mathrm{~m} / \mathrm{s}$ was thus chosen. Using a wavelength of $4.77 \pm 0.2 \mathrm{~mm}$ determined from the summed power spectrum (giving $\lambda / \delta=2.06$ ), a frequency of 409 $\pm 20 \mathrm{kHz}$ is obtained. The uncertainty in the wavelength is estimated here from the width of the spectrum peak at $1 / \mathrm{e}$ times the maximum. We note that the camera frame-rate employed, $500 \mathrm{kHz}$, is barely high enough to allow the unambiguous interpretation of results at such conditions. A local linear stability analysis of this experiment, assuming a perfect gas, was performed using the NOLOT code ${ }^{14}$ and a most-amplified frequency of $434 \mathrm{kHz}$ at the relevant position downstream was obtained.

This experimental analysis was repeated for the other image sequence in which boundary-layer waves were observed. Two dominant wavelengths were seen in the power spectra: the reason for this is not entirely clear, though we note that the disturbances here were weaker than in the previous sequence, which may affect the accuracy of the derived wavelengths. Corresponding frequencies of 263 and $315 \mathrm{kHz}$ were deduced; the most-amplified frequency obtained from NOLOT was $325 \mathrm{kHz}$. The computational results for both runs are presented in Figure 4 in the form of neutral stability diagrams. The experimentally measured wave frequency in each case (assuming the higher frequency in the latter experiment to be representative) is seen to lie well within the corresponding computational region of positive amplification. The agreement between experimentally measured and computational mostamplified frequencies observed here is similar to that obtained in Ref. 15 (2-14\%) under less challenging experimental conditions. It should be pointed out, however, that the NOLOT computations were based on the nozzle exit conditions: the Reynolds number at the cone tip was slightly higher, and the boundary layer might have retained some memory of this at the visualized position downstream, which would shift the computational frequencies to slightly higher values.

Additionally, the wave "structure angle"10, i.e., the inclination of lines of constant phase in the $x-y$ plane, was determined by one-dimensional spatial correlations between neighboring rows in a single image. At $y / \delta=0.4$, the measured angle is $24^{\circ}$, which then decreases to $14-15^{\circ}$ between $y / \delta=0.6$ and 0.7 . These results are consistent with the hot-film measurements of Ref. 10, though no assumption regarding the convection velocity needs to be made here. 
However, instead of the structure angle increasing to $50-60^{\circ}$ as $y / \delta$ is subsequently increased, here a further drop in angle is observed, consistent with the turning over of the waves seen in the images. This may be an artifact of the Schlieren technique, as the horizontal orientation of the knife edge means that features forming a large angle with the cone surface will not be well-resolved; this may also be the reason that disturbances are not observed to extend past the boundary-layer edge in Figure 3. This limitation could be addressed by the use of a circular cutoff; regardless, as the transition process is driven by the larger relative disturbance amplitudes further inside the boundary layer, being unable to measure near the boundary-layer edge is of limited practical significance.

Finally, we return to the issue of the inability of standard Schlieren techniques to resolve the spanwise dependence of the second-mode disturbance profile. Assuming the spanwise extent of the disturbances to be limited to a few boundary-layer thicknesses ${ }^{10}$, this inability means the $y / \delta$ dependence of the signal also cannot be determined, unless the spanwise location of the disturbance can be otherwise resolved. One way this could be achieved is through a spanwise array of surface-mounted sensors at the visualized location downstream. A second approach would be to employ a focused Schlieren system, as in Ref. 9. The depth-of-focus in this setup was approximately $1 \mathrm{~cm}$, sufficiently small to isolate disturbances in the spanwise direction (for which a rough criterion is that the depth-of-focus must be much less than $\sqrt{2 \delta r}$, where $r$ is the radius of the cone). However, focused Schlieren systems are known to be generally less sensitive and more difficult to implement than standard Schlieren setups $^{16}$. A third option is to introduce artificial disturbances ${ }^{17,18}$, allowing the spanwise location of the instability waves to be controlled.

\section{Conclusions}

The preceding measurements indicate that it is possible to derive structural and propagation characteristics of boundary-layer instability waves from high-speed Schlieren cinematography; future experiments will help to clarify whether such measurements can be routinely obtained. The determination of spatial amplification rates would be significantly more challenging, though may be possible using a well calibrated setup with uniform illumination. It should be noted, however, that due to their large sensing heads, pressure transducers are also questionable with regard to their ability to measure amplification rates. 


\begin{tabular}{|l|l|l|l|l|l|l|l|l|l|}
\hline Condition & $\mathrm{p}_{0}, \mathrm{MPa}$ & $\mathrm{h}_{0}, \mathrm{MJ} \mathrm{kg}^{-1}$ & $\mathrm{r}_{\mathrm{n}}, \mathrm{mm}$ & $\mathrm{Re}, \mathrm{x} 10^{6} \mathrm{~m}^{-1}$ & $\mathrm{M}_{\infty}$ & $\mathrm{u}_{\infty}, \mathrm{m} \mathrm{s}^{-1}$ & $\mathrm{~T}_{\infty}, \mathrm{K}$ & $\mathrm{p}_{\infty}, \mathrm{Pa}$ & $\rho_{\infty}, \mathrm{kg} \mathrm{m}^{-3}$ \\
\hline $\mathrm{I}$ & 28.5 & 3.4 & 5 & 6.2 & 7.3 & 2370 & 266 & 3690 & 0.0481 \\
\hline II & 15.5 & 3.4 & 5 & 3.4 & 7.3 & 2380 & 261 & 1870 & 0.0246 \\
\hline
\end{tabular}

Table 1: Computed test conditions for the two experiments described herein: $p_{0}$ and $h_{0}$ are the reservoir pressure and enthalpy, $\mathbf{r}_{\mathrm{n}}$ is the cone nose radius, $R e$ is the free-stream unit Reynolds number, and $\mathbf{M}_{\infty}, \mathbf{u}_{\infty}, \mathbf{T}_{\infty}$, $p_{\infty}$, and $\rho_{\infty}$ are the free-stream Mach number, velocity, temperature, pressure and density, respectively.
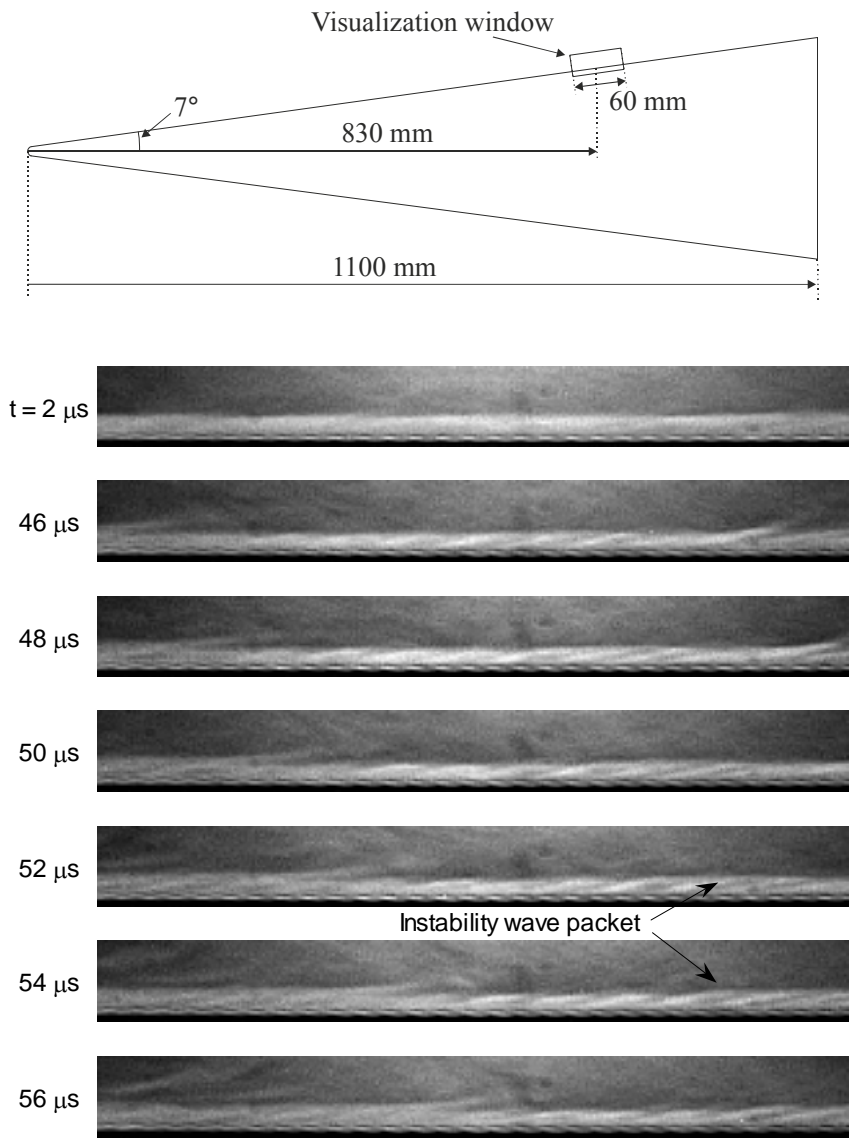

Fig. 1 (Above) Schematic of the cone model and the visualization region; (below) sequence showing the development of an instability wave packet (46-56 us) within the boundary layer. The regular structures visible in the two rows of pixels immediately above the cone surface are artifacts of the image-processing techniques employed. 


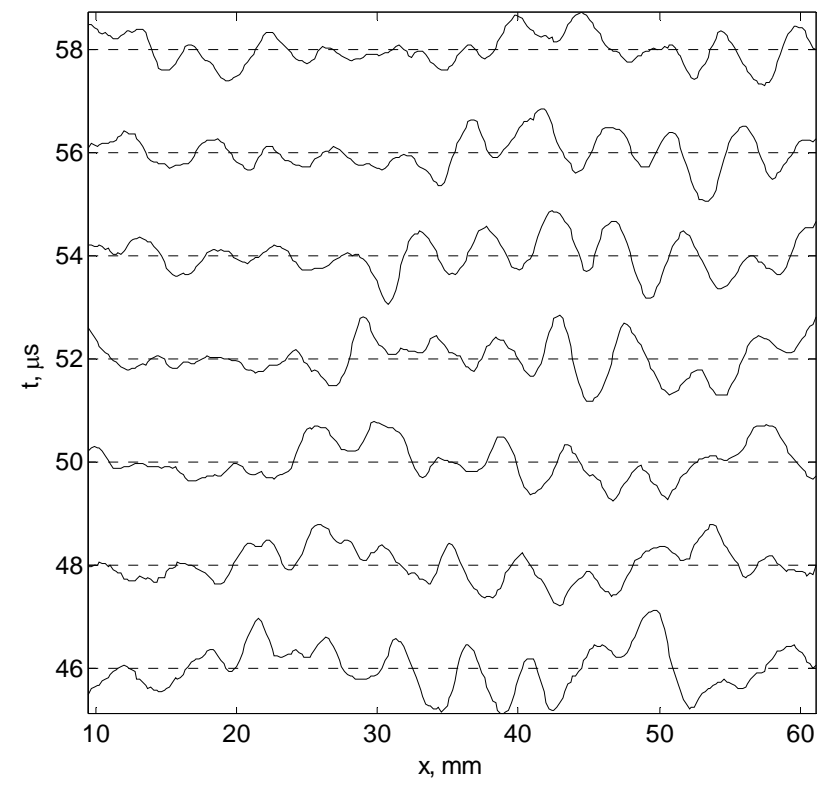

Fig. $2 \mathrm{X}$-t diagram showing the development of the disturbance waves seen in Fig. 1 at $y / \delta \approx 0.5$; the plotted value is the normalized pixel intensity.

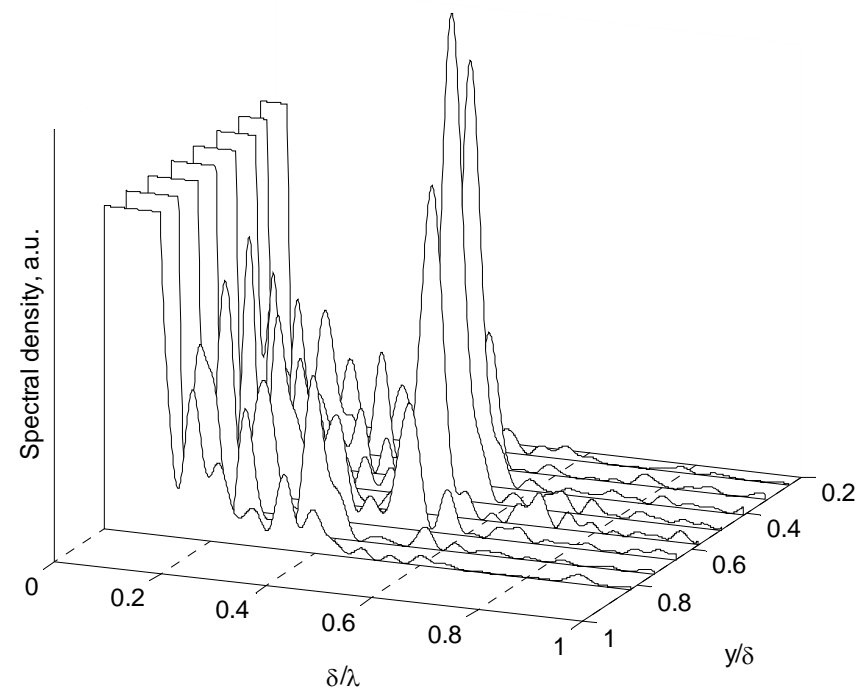

Fig. 3 Energy spectral density as a function of normalized inverse wavelength (i.e., frequency) for various heights above the cone surface at $\mathrm{t}=54 \mu \mathrm{s}$. 


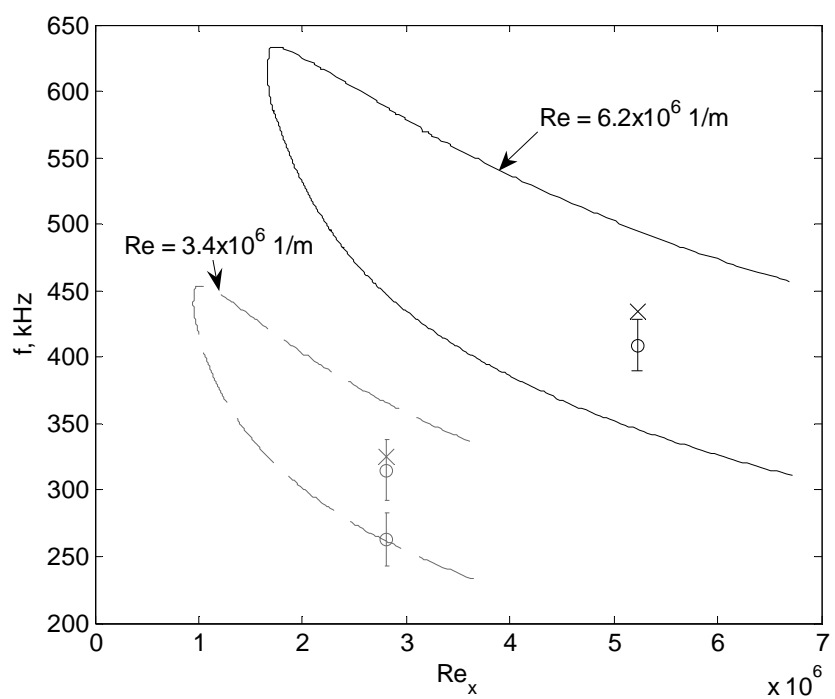

Fig. 4 Computational neutral stability diagrams for the two experiments analyzed in this work. The circles indicate the measured wave frequencies, while the cross in each case indicates the computational mostamplified frequency at the measured point downstream.

\section{Acknowledgments}

The authors wish to acknowledge insightful comments from Hans G. Hornung.

\section{References}

[1] Germain, P. D., and Hornung, H. G., “ Transition on a Slender Cone in Hypervelocity Flow,” Experiments in Fluids, Vol. 22, 1997, pp. 183-190.

[2] Johnson, H. B., Seipp, T. G., and Candler, G. V., "Numerical Study of Hypersonic Reacting Boundary Layer Transition on Cones," Physics of Fluids, Vol. 10, No. 10, 1998, pp. 2676-2685.

[3] Reshotko, E., "Boundary-Layer Stability and Transition,” Annual Review of Fluid Mechanics, Vol. 8, 1975, pp. 311-349.

[4] Schneider, S. P., "Effects of High-Speed Tunnel Noise on Laminar-Turbulent Transition,” Journal of Spacecraft and Rockets, Vol. 38, No. 3, 2001, pp. 323-333.

[5] Tanno, H., Komuro, T., Sato, K., Itoh, K., Takahashi, M., and Fujii, K., "Measurement of Hypersonic Boundary Layer Transition on Cone Models in the Free-Piston Shock Tunnel HIEST,” AIAA Paper 2009-781, Jan. 2009.

[6] Maslov, A. A., Fedorov, A. V., Bountin, D. A., Shiplyuk, A. N., Sidorenko, A. A., Malmuth, N. D., and Knauss, H., "Experimental Study of Disturbances in Transitional and Turbulent Hypersonic Boundary Layers,” AIAA Journal, Vol. 46, No. 7, 2008, pp. 1880-1883. 
[7] Fischer, M. C., and Wagner, R. D., "Transition and Hot-Wire Measurements in Hypersonic Helium Flow," AIAA Journal, Vol. 10, No. 10, 1972, pp. 1326-1332.

[8] Demetriades, A., "Hypersonic Viscous Flow Over a Slender Cone, Part III: Laminar Instability and Transition,” AIAA Paper 74-535, June 1974.

[9] VanDercreek, C. P., Smith, M. S., and Yu, K. H., "Focused Schlieren and Deflectrometry at AEDC Hypervelocity Wind Tunnel No. 9,” AIAA Paper 2010-4209, June 2010.

[10] Kimmel, R. L., Demetriades, A., and Donaldson, J. C., "Space-Time Correlation Measurements in a Hypersonic Transitional Boundary Layer," AIAA Journal, Vol. 34, No. 12, 1996, pp. 2484-2489.

[11] Wagner, A., Laurence, S., Martinez Schramm, J., Hannemann, K., Wartemann, V., Luedeke, H., Tanno, H., and Ito, K., "Experimental Investigation of Hypersonic Boundary-Layer Transition on a Cone Model in the High Enthalpy Shock Tunnel (HEG) at Mach 7.5,” AIAA Paper 2011-2374, April 2011.

[12] Kroll, N., and Fassbender, J.K., "MEGAFLOW - Numerical Flow Simulation for Aircraft Design”, Springer, Berlin, 2005.

[13] Stetson, K. F., and Kimmel, R. L., “On Hypersonic Boundary-Layer Stability,” AIAA Paper 92-0737, Jan. 1992.

[14] Hein, S., Bertolotti, F.P., Simen, M., Hanifi, A., and Henningson, D., "Linear nonlocal instability analysis: the linear NOLOT code," DLR-IB 223-94 A56, 1994.

[15] Alba, C.R., Casper, K.M., Beresh, S.J., and Schneider, S.P., "Comparison of Experimentally Measured and Computed Second-Mode Disturbances in Hypersonic Boundary-Layers,” AIAA Paper 2010-897, Jan. 2010.

[16] Settles, G., “Schlieren and shadowgraph techniques,”.Springer, NY, 2006.

[17] Maslov, A. A., Shiplyuk, A.,N., Sidorenko, A. A., and Arnal, D., "Leading-Edge Receptivity of a Hypersonic Boundary Layer on a Flat Plate,” Journal of Fluid Mechanics, Vol. 426, 2001, pp. 73-94.

[18] Heitmann, D., Kähler, C., Radespiel, R., Rödiger, T., Knauss, H., and Wagner, S., „Non-Intrusive Generation of Instability Waves in a Planar Hypersonic Boundary Layer,” Experiments in Fluids, Vol. 50, 2011, pp. 457-464. 